SU-ITP-97-39, hep-th/9709202

\title{
Quantization of p-branes, D-p-branes and M-branes *
}

\author{
R. Kallosh \\ Physics Department, Stanford University, Stanford, CA 94305-4060
}

Killing spinors of space-time BPS configurations play an important role in quantization of theories with the fermionic worldvolume local symmetry. We show here how it works for the GS superstring, BST supermembrane and M-5-brane. We show that the non-linear generalization of the $(2,0) \mathrm{d}=6$ tensor supermultiplet action is the M-5-brane action in a Killing gauge. For D-p-branes the novel feature of quantization is that they can be quantized Lorentz covariantly, in particular, for D-0-brane a gauge exists where the action is covariant and free. We present a general condition on possible choice of gauges for the $\kappa$-symmetric branes.

We review here some work on quantization of the local fermionic worldvolume symmetry presented in 114. This is a generalized version of the talk presented at Strings 97 in Amsterdam in June 1997. We give a short summary of the new results in the end of this paper.

\section{WORLDVOLUME ACTIONS AND THEIR LOCAL SYMMETRIES}

The actions of Green-Schwarz string in $\mathrm{D}=10$ target space and of the Bergshoeff-SezginTownsend membrane in $\mathrm{D}=11$ target space have local fermionic $\kappa$-symmetry, reparametrization invariance and manifest space-time supersymmetry. They depend on worldvolume fields $Z^{M}=\left(X^{m}(\xi), \theta^{\alpha}(\xi)\right)$ related to coordinates of the space-time superspace. Here $X^{m}, m=$ $0,1, \ldots, D-1$ are bosonic and $\theta^{\mu}, \mu=1, \ldots, 32$ are fermionic coordinates of the superspace. The bosonic coordinates on the brane are $\xi^{i}, i=$ $0, \ldots, p$. For the string $p=1$ and for the membrane $p=2$. Both actions belong to a general class of $\kappa$-symmetric p-brane actions and upon gauge-fixing describe the scalar supermultiplets of worldvolume supersymmetry.

\footnotetext{
*To appear in the Proceedings of Strings 97, Amsterdam, June 1997. This work is supported by the NSF grant PHY9219345 and a NATO Collaboration Research Grant.
}

D-p-brane actions [0] depend in addition on world-volume 1-form gauge potential $A_{i}$ and upon gauge-fixing describe the vector multiplets of worldvolume supersymmetry.

Finally, the M-5-brane action [8,9] depends on a 2-form gauge potential $A_{i j}$ with the self-dual field strength. This action after gauge-fixing describes the tensor multiplets of worldvolume supersymmetry.

The class of actions we consider have 32dimensional global space-time supersymmetry and the local fermionic $\kappa$-supersymmetry :

$$
\begin{aligned}
& \delta_{\epsilon} \theta=\epsilon, \quad \delta_{\epsilon} X^{m}=\bar{\epsilon} \Gamma^{m} \theta, \\
& \delta_{\kappa} \theta=(1+\Gamma) \kappa(\xi), \\
& \delta_{\kappa} X^{m}=\bar{\theta} \Gamma^{m} \delta \theta_{\kappa}, \ldots .
\end{aligned}
$$

Both $\epsilon$ and $\kappa$ are 32-dimensional, but $\epsilon$ is global and $\kappa$ depends on coordinates of the brane $\xi$. Here dots mean the transformations of BornInfeld or a tensor field. $\Gamma$ is a function of the fields of the brane $\Gamma=\Gamma(Z(\xi), A(\xi))$ and depends on $\xi^{i}$ therefore. The matrix $\Gamma(\xi)$ squares to 1 and has a vanishing trace: therefore $(1+\Gamma)(1-\Gamma)=0$, i.e. $1+\Gamma$ is a projector which makes a 32 -dimensional parameter of $\kappa$-supersymmetry effectively only 16-dimensional. This allows to exclude half of the 
original $\theta$ variables in the classical action from the quantized action.

Local reparametrization symmetry of the worldvolume includes transformations of the type $\delta Z^{M}(\xi)=\delta \xi^{i} \partial_{i} Z^{M}(\xi)$ for the scalars on the worldvolume, etc. Here $\delta \xi^{i}(\xi)$ depends on coordinates of the worldvolume, i.e. it is a gauge symmetry, which allows us to exclude some bosonic fields from the classical action.

Quantization, or gauge-fixing of local symmetries of the worldvolume actions will be described here in detail in a Killing adapted gauge but also in most general gauges [4]. The local symmetries will be used to exclude the unphysical degrees of freedom. The resulting worldvolume actions have some particular form of the global supersymmetry, which is a combination of the original space-time supersymmetry and a special choice of $\kappa$-symmetry which preserves the Killing spinor adapted gauge. The advantage of using different choice of gauges will be emphasized [3].

\section{KILLING SPINOR ADAPTED GAUGE}

We define the space-time Killing spinor adapted gauge as follows. First we define an irreducible $\kappa$-symmetry with only 16 parameters by imposing a condition which $\kappa$ has to satisfy.

$\mathcal{P}_{-} \kappa=\frac{1}{2}(1-\gamma) \kappa=0$

Now we can use this 16-dimensional local fermionic symmetry to eliminate 16 of $\theta^{\prime} s$.

$\mathcal{P}_{+} \theta=\frac{1}{2}(1+\gamma) \theta=0$

The choice of the $\xi^{i}$-independent projectors $\mathcal{P}_{ \pm}=\frac{1}{2}(1 \pm \gamma)$ which can divide a 32-dimensional spinor into 2 parts is made with the help of a Killing spinor of the space-time configuration describing a particular extended object which we are quantizing here. This means that

$\gamma=\left.\Gamma\right|_{\mathrm{cl}}$

i.e. the constant projector $(1+\gamma)$ can be chosen as the $\kappa$-symmetry projector $(1+\Gamma)$ taken at the values of fields which form a classical solution describing the relevant bosonic brane. For example, the classical solution for the p-brane is

$X^{i}=\xi^{i}, \quad X^{a^{\prime}}=$ const,$\quad \theta=0$

We have split here the bosonic coordinate of the space-time $X^{m}$ into the part $X^{i}$ which is identified with the bosonic coordinate of the brane $\xi^{i}$ and the coordinates $X^{a^{\prime}}$ which are transverse to the brane which will become the worldvolume scalars. In the classical solution identifying the Killing spinor of the space-time those are taken to be constants.

The Killing spinor of a space-time geometry naturally can not depend on the coordinates of the worldvolume.

In a basis in which $\gamma$ is diagonal all spinors split accordingly:

$$
\theta=\left(\begin{array}{c}
\theta^{\alpha} \\
\theta^{\alpha^{\prime}}
\end{array}\right), \quad \kappa=\left(\begin{array}{c}
\kappa^{\alpha} \\
\kappa^{\alpha^{\prime}}
\end{array}\right), \quad \epsilon=\left(\begin{array}{c}
\epsilon^{\alpha} \\
\epsilon^{\alpha^{\prime}}
\end{array}\right)
$$

In this basis the space-time Killing spinor adapted gauge (3), (4) takes a simple form

$\kappa^{\alpha^{\prime}}=0, \quad \theta^{\alpha}=0$

In the same basis the field dependent $\kappa$ symmetry generator $\Gamma$ has the following block structure

$\Gamma=\left(\begin{array}{cc}+C & \left(1-C^{2}\right) A^{-1} \\ A & -C\end{array}\right)$,

where the $16 \times 16$ dimensional matrices $C$ and $A$ commute, $A C-C A=0$. The matrices $1 \pm \Gamma$ have vanishing determinants and rank 16 , which means that the 16 -dimensional matrices $1 \pm C$ and $A$ are invertible.

If some choice of a projector $\mathcal{P}_{ \pm}$leads to noninvertible $\mathrm{A}$ or $1 \pm C$, this projector can not be used for quantization. Examples include $\mathrm{d}=10$ Lorentz covariant gauges for the Green-Schwarz string.

Consider now a combination of 16-dimensional irreducible $\kappa$-symmetry and 32-dimensional space-time supersymmetry in our gauge

$$
\begin{aligned}
\delta_{\kappa, \epsilon} \theta^{\alpha} & =(1+C)^{\alpha}{ }_{\beta} \kappa^{\beta}+\epsilon^{\alpha}=0 \\
\delta_{\kappa, \epsilon^{\prime}} \theta^{\alpha^{\prime}} & =A^{\alpha^{\prime}}{ }_{\beta} \kappa^{\beta}+\epsilon^{\alpha^{\prime}}
\end{aligned}
$$


One has to impose the relation between the parameters of local $\kappa$-symmetry and global spacetime supersymmetry $\epsilon$ which will keep the gauge $\theta^{\alpha}=0$. It is obtained from eq. (9) under condition that the matrix $(1+C)$ is invertible.

$\kappa^{\beta}(\xi)=-\left[(1+C)^{-1}\right]^{\beta}{ }_{\alpha} \epsilon^{\alpha}$

Note that the matrix $C$ is a function of the fields of the theory and therefore depends on the coordinated of the brane.

\section{WORLDVOLUME SUPERSYMME- TRY ON THE BRANE}

Thus we have explicitly gauge-fixed so far only the fermionic symmetry by requiring $\theta^{\alpha}=0$. In what follows we will also specify the gauge-fixing of the reparametrization symmetry by choosing the so-called static gauge. This gauge may also be qualified as a Killing vector adapted gauge, as the directions along the branes are in fact related to Killing vectors of the space-time brane configuration.

$X^{i}(\xi)-\xi^{i}=0$

If the reparametrization symmetry is fixed by choosing a static gauge, the space-time spinors (former scalars on the worldvolume) like $\theta^{\alpha^{\prime}}$ and $\epsilon^{\alpha}, \epsilon^{\alpha^{\prime}}$ become worldvolume spinors.

To extract the 32-dimensional global supersymmetry transformation of $16 \theta^{\alpha^{\prime}}$ living on the brane we have to insert the value of the $\kappa$-symmetry parameter transformations (11) which keeps the $\theta^{\alpha}=0$ gauge into eq. (10)

$\delta_{\epsilon, \epsilon^{\prime}} \theta^{\alpha^{\prime}}=\left(-A^{\alpha^{\prime}}{ }_{\beta}\left[(1+C)^{-1}\right]_{\alpha}^{\beta} \epsilon^{\alpha}\right)_{\mathrm{cl}}+\epsilon^{\alpha^{\prime}}$

This is the general answer 何. The subscript ()$_{\mathrm{cl}}$ means that the values of the matrices $A, C$ have to be taken at the classical values of the brane fields as given e. g. in eqs. (6).

Given a $\kappa$-symmetry of the action is known and the right choice of the constant projector is made, which supplies us with $16 \times 16$ matrices $C$ and $A$, we have the answer for the worldvolume supersymmetry. The meaning of the matrices $A$ and $C$ can be understood if we represent the full generator $\Gamma$ as consisting of 2 parts $\Gamma=\Gamma_{A}+\Gamma_{C}$ where $\Gamma_{A}$ anticommutes with the projector $\gamma$ and $\Gamma_{C}$ commutes with it.

$$
\Gamma_{A}=\left(\begin{array}{cc}
0 & \left(1-C^{2}\right) A^{-1} \\
A & 0
\end{array}\right),
$$

and

$$
\Gamma_{C}=\left(\begin{array}{cc}
+C & 0 \\
0 & -C
\end{array}\right) .
$$

This is in the basis where

$$
\gamma=\left(\begin{array}{cc}
1 & 0 \\
0 & -1
\end{array}\right)
$$

Thus

$$
\left\{\gamma, \Gamma_{A}\right\}=0, \quad\left[\gamma, \Gamma_{C}\right]=0 .
$$

The condition for the gauge-fixing to be admissible and to produce an action with global supersymmetry is that $A$ and $1 \pm C$ are invertible.

\section{EXAMPLES}

\subsection{GS Superstring}

GS superstring belongs to a class of $\kappa$ symmetric p-brane actions. The $\kappa$-symmetry generator is

$$
\Gamma=\frac{1}{2 ! \sqrt{-g}} \epsilon^{i j} \gamma_{i j}
$$

Here $\gamma_{i}=\Pi_{i}{ }^{m} \Gamma_{m}$ and $\Pi_{i}{ }^{m}=\partial_{i} X^{m}-\bar{\theta} \Gamma^{m} \partial_{i} \theta$. We will make a split $m=i, a^{\prime}$ where $i=0,9$ and $a^{\prime}=1, \ldots, 8$. The Killing spinor gauge for the fundamental string is in fact exactly the lightcone gauge for the spinors. Indeed we take as a classical solution

$X^{0}=\tau, \quad X^{9}=\sigma, X^{a^{\prime}}=$ const,$\quad \theta=0$

and the $\kappa$-symmetry generator at this point in configuration space gives us a projector $1+\gamma=$ $1+\left.\Gamma\right|_{\mathrm{cl}}=1+\Gamma_{09}$, Thus we have to require that

$\left(1+\Gamma_{09}\right) \theta=0 \quad\left(1-\Gamma_{09}\right) \kappa=0$

which is equivalent to the standard light-cone gauge for the spinors

$\Gamma^{+} \theta=0 \quad \Gamma^{-} \kappa=0$ 
We may in addition choose the static gauge for the string $X^{0}=\tau, \quad X^{9}=\sigma$. If we start with the version of the theory where the worldsheet metric is the induced one, we will bring the theory to the form of a 2-dimensional nonlinear action of the scalar multiplet which depends on 82 -dimensional scalars and 8 physical spinors. The amount of unbroken supersymmetries is 32 . To find out the supersymmetry transformations of the spinors $\Gamma^{-} \theta=\theta^{\alpha^{\prime}}$ we have to find out the relevant $A$ and $C$ matrices. Clearly, $\Gamma_{i j}, \Gamma_{a^{\prime} b^{\prime}}$ commute with $\Gamma_{i j}$ and $\Gamma_{i a^{\prime}}$ anticommute. In Killing spinor and vector adapted gauge $\Pi_{i}{ }^{j}=\delta_{i}{ }^{j}-\bar{\theta} \Gamma^{j} \partial_{i} \theta$ and $\Pi_{i}{ }^{a^{\prime}}=\partial_{i} X^{a^{\prime}}$. This gives us the presentation of the full $\Gamma$ in terms of commuting and anticommuting parts: for $\Gamma_{C}$ we find

$$
\begin{gathered}
\epsilon^{i j}\left(\delta_{i}^{i^{\prime}}-\bar{\theta} \Gamma^{i^{\prime}} \partial_{i} \theta\right)\left(\delta_{j} j^{j^{\prime}}-\bar{\theta} \Gamma^{j^{\prime}} \partial_{j} \theta\right) \Gamma_{i^{\prime} j^{\prime}}+ \\
\epsilon^{i j} \partial_{i} X^{a^{\prime}} \partial_{j} X^{b^{\prime}} \Gamma_{a^{\prime} b^{\prime}}
\end{gathered}
$$

and $\Gamma_{A}$ is

$$
\epsilon^{i j}\left(\delta_{i}^{i^{\prime}}-\bar{\theta} \Gamma^{i^{\prime}} \partial_{i} \theta\right) \partial_{j} X^{b^{\prime}} \Gamma_{i^{\prime} b^{\prime}}
$$

Thus the global non-linear worldvolume supersymmetry transformation for the GS superstring quantized in the Killing adapted gauge is given by eq. (13) where the matrices $A, C$ are defined in eqs. above and depend on scalars $X^{a^{\prime}}$ and spinors $\theta^{\alpha^{\prime}}$.

\subsection{BST supermembrane}

BST supermembrane also belongs to a class of $\kappa$-symmetric p-brane actions. The $\kappa$-symmetry generator is

$$
\Gamma=\frac{1}{3 ! \sqrt{-g}} \epsilon^{i j k} \Gamma_{i j k}
$$

To find the Killing spinor adapted gauge for the supermembrane we first consider the generator above taken at

$X^{0}=\tau, \quad X^{9}=\sigma, \quad X^{10}=\rho$

and

$X^{a^{\prime}}=$ const,$\quad \theta=0$

Thus we get

$\gamma=\Gamma_{0} \Gamma_{9} \Gamma_{10}$
We see that for the membrane as different from the string the light-cone gauge is not a natural one, as there are 3 Killing directions, time and 2 more and not just one as in case of the string. Thus the Killing gauge for the supermebrane is

$\left(1+\Gamma_{0} \Gamma_{9} \Gamma_{10}\right) \theta=0, \quad\left(1-\Gamma_{0} \Gamma_{9} \Gamma_{10}\right) \kappa=0$

and

$X^{0}=\tau, \quad X^{9}=\sigma, \quad X^{10}=\rho$

The field remaining in the gauge-fixed action are the scalars

$$
X^{a^{\prime}}(\tau, \sigma, \rho)
$$

and the fermions

$$
\left(1-\Gamma_{0} \Gamma_{9} \Gamma_{10}\right) \theta(\tau, \sigma, \rho) .
$$

The procedure of extracting the full non-linear 3-dimensional action of the scalar supermultiplet follows as explained above. The action is given by the supermebrane action (in a version of the supermembrane theory with the metric induced on the brane, not independent variable) at the surface defined by the Killing gauge. For the global supersymmetry we have to find $A, C$ parts of $\Gamma$ and use eq. (13).

It is useful to point out here that the complete and detailed quantization of the supermebrane was performed so far only in the light-cone gauge for spinors.

\subsection{M-5-brane and $(0,2)$ tensor supermul- tiplet in $d=6$}

We will give here a brief description of gaugefixing procedure of the M-5-brane theory which provides the supersymmetric action for $(0,2)$ tensor supermultiplet in $\mathrm{d}=6$. The manifestly $\mathrm{d}=6$ general coordinate invariant $\mathrm{M}-5$-brane action was found by I. Bandos, K. Lechner, A. Nurmagambetov, P. Pasti, D. Sorokin and M. Tonin [8]

$S_{M-5}\left(X^{m}(\xi), \theta^{\mu}(\xi), A_{j k}(\xi), a(\xi)\right)$.

The action depends on $Z^{M}$, the metric, induced on the brane, on the tensor field with the self-dual field strength and auxiliary worldvolume scalar field $a(\xi)$ which serves to achieve the manifestly 
$d=6$ general coordinate invariance of the M-5brane action.

The $\kappa$-symmetry transformations and supersymmetry of space-time fermions are

$\delta_{\kappa, \epsilon} \theta=(1+\Gamma) \kappa+\epsilon$

where

$\Gamma=\Gamma_{(0)}+\Gamma_{(3)}$

and

$\begin{aligned} \Gamma_{(0)} & =\frac{1}{6 ! \sqrt{|g|}} \epsilon^{i_{1} \cdots i_{6}} \gamma_{i_{1}} \cdots \gamma_{i_{6}} \\ \Gamma_{(3)} & =\frac{1}{2 \cdot 3 !} h_{i j k} \gamma^{i j k}\end{aligned}$

Here $i=0, \ldots, 5, \quad m=0, \ldots, 10$.

Here we are using the form of $\kappa$-symmetry transformations found originally in the superembedding approach by P.S. Howe, E. Sezgin and P.C. West [10] and proved later to be also a symmetry transformation of the M-5-brane action in [11. The worldvolume field $h_{i j k}$ of [10] turns out to be a non-linear function of the fields in the action, whose explicit form can be found in [11].

The gauge-fixing of the M-5-brane action is inspired by the superembedding [10] of the spacetime superspace with coordinates $X^{m}, \Theta^{\mu}$ into worldvolume superspace with coordinates $\xi^{i}, \theta^{\alpha}$. We split $m=\left(i, a^{\prime}\right), \mu=\left(\alpha, \alpha^{\prime}\right)$. The superembedding is $X^{i}=\xi^{i}, \Theta^{\alpha}=\theta^{\alpha}$ and $X^{a^{\prime}}=$ $X^{a^{\prime}}(\xi, \theta), \Theta^{\alpha^{\prime}}=\Theta^{\alpha^{\prime}}(\xi, \theta)$. To be as close to this as possible in the bosonic action of the 5-brane we have to require that in our action

$X^{i}=\xi^{i}, \theta^{\alpha}=0$

and the fields of the $(0,2)$ tensor multiplet remaining in the action which depend on $\xi$ are

$$
\begin{aligned}
& X^{a^{\prime}}(\xi), \theta^{\alpha^{\prime}}(\xi), A_{i j}(\xi), a(\xi), \\
& \quad a^{\prime}=1,2,3,4,5, \quad \alpha^{\prime}=1,2, \ldots, 16 .
\end{aligned}
$$

Thus we have 5 scalars $X^{a^{\prime}}(\xi)$, a 16-component spinor $\theta^{\alpha^{\prime}}(\xi)$ which can considered (see below) as a a chiral $\mathrm{d}=6$ spinor with a $U S p(4)$ symplectic Majorana-Weyl reality condition $\theta_{s}^{\hat{\alpha}}$, a tensor $A_{i j}(\xi)$ with the self-dual field strength and an auxiliary scalar $a(\xi)$. The $11 \mathrm{~d} 32 \times 32 \Gamma^{m}$ matrices have to be taken in the basis which correspond to the split of the target superspace into the superspace of the 5-brane and the rest [10]. This reflects the $\operatorname{Spin}(1,5) \times U S p(4)$ symmetry of the six dimensional theory. An 11d Majorana spinor decomposes as $\psi=\left(\psi_{\hat{\alpha} s}, \psi_{s}^{\hat{\alpha}}\right)$ where $s=1,2,3,4$ is an $U S p(4)$ index and $\hat{\alpha}=1,2,3,4$ is a $6 \mathrm{~d}$ Weyl spinor index with upper (lower) indices corresponding to anti-chiral (chiral) spinors respectively. The $6 \mathrm{~d}$ spinors satisfy a MajoranaWeyl reality condition. The relevant representation of $11 \mathrm{~d} \Gamma^{m}$ is $\Gamma_{\hat{\alpha} s, \hat{\beta} t}^{i}=\eta_{s t}\left(\sigma^{i}\right)_{\hat{\alpha} \hat{\beta}}$ where $\eta_{s t}$ is the $U S p(4)$ antisymmetric invariant metric and $\sigma^{i}$ the $6 \mathrm{~d}$ chirally-projected gamma-matrices etc. [10]. In terms of 16-component spinors we have $\psi_{\hat{\alpha} s}=\psi^{\alpha^{\prime}}, \psi_{s}^{\hat{\alpha}}=\psi^{\alpha}$.

Thus we choose a projector $\gamma$ to be a chiral projector of the 6-dimensional space times the unit matrix. In the basis above this means that

$\gamma=\left.\Gamma\right|_{c l}=\left.\frac{1}{6 !} \epsilon^{i_{1} \cdots i_{6}} \gamma_{i_{1}} \cdots \gamma_{i_{6}}\right|_{c l}=\left(\begin{array}{cc}1 & 0 \\ 0 & -1\end{array}\right)$

Here the subscript $c l$ means that we take $X_{c l}^{a^{\prime}}=$ const, $\theta_{c l}=0,\left(h_{i j k}\right)_{c l}=0,\left(E_{i}{ }^{j}\right)_{c l}=$ $\delta_{i}{ }^{j},\left(E_{i}{ }^{a^{\prime}}\right)_{c l}=0$ and the field-independent part of the $\kappa$-symmetry generator $1+\Gamma$ provides us with the projector for gauge-fixing $\kappa$-symmetry. Note that this is exactly the projector which specifies the M-5-brane Killing spinor in the target space.

The gauge fixed theory is given by the classical action in the gauge (27). Since both the reparametrization symmetry and $\kappa$-symmetry are fixed in a unitary way, there are no propagating ghosts. The action for a tensor multiplet is an action of a M-5 brane in a Killing spinor adapted gauge with vanishing $X^{i}(\xi)-\xi^{i}$ and $\theta^{\alpha}(\xi)$ :

$$
\begin{gathered}
S_{(0,2)}\left(X^{a^{\prime}}(\xi), \theta^{\alpha^{\prime}}(\xi), A_{j k}(\xi), a(\xi)\right)= \\
S_{M-5}\left(X^{a^{\prime}}, \theta^{\alpha^{\prime}} A_{j k}, a, X^{i}=\xi^{i}, \theta^{\alpha}=0\right) .
\end{gathered}
$$

To find the exact non-linear worldvolume supersymmetry transformations of the $S_{(0,2)}$ action we may now proceed using the rules from the previous section. In addition to gauge fixing the 
spinor theta we have to gauge fix the infinite reducible $\kappa$-symmetry. We choose as before

$\theta^{\alpha}=0, \quad \kappa^{\alpha^{\prime}}=0$

To extract from the generator of $\kappa$-symmetry $\Gamma$ the matrices $C$ and $A$ which define the worldvolume supersymmetry we have to take into account that in the flat 11-dimensional background

$$
\begin{aligned}
\Gamma & =\frac{1}{6 ! \sqrt{|g|}} \epsilon^{i_{1} \cdots i_{6}}\left(\gamma_{i_{1}} \cdots \gamma_{i_{6}}+40 \gamma_{i_{1}} \gamma_{i_{2}} \gamma_{i_{3}} h_{i_{4} i_{5} i_{6}}\right) \\
\gamma_{i} & =\left(\delta_{i}{ }^{j}-i \bar{\theta} \Gamma^{j} \partial_{i} \theta\right) \Gamma_{j}+\partial_{i} X^{a^{\prime}} \Gamma_{a^{\prime}} .
\end{aligned}
$$

Here we used the fact that the spinors are chiral and therefore $\bar{\theta} \Gamma^{a^{\prime}} \partial_{i} \theta$ vanishes. Using eq. (31) we may rewrite $\Gamma$ as a sum of products of $\Gamma$ 's

$$
\Gamma=\sum_{n} \Gamma^{i_{1}} \cdots \Gamma^{i_{n}} F_{i_{i} \cdots i_{m}}\left(X^{a^{\prime}}, \theta^{\alpha^{\prime}}, h_{i j k}\right)
$$

All terms with even number $n=2,4,6$ of $6 \Gamma^{i}$ will contribute only to $C$, all terms with odd number $n=1,3,5$ of $\Gamma^{i}$ will contribute to $A$ since $\Gamma^{i}$ is off-diagonal in our basis. The dependence on diagonal matrices

$$
\sum_{n=2,4,6} \Gamma^{i_{1}} \cdots \Gamma^{i_{n}} F_{i_{i} \cdots i_{m}}\left(X^{a^{\prime}}, \theta^{\alpha^{\prime}}, h_{i j k}, a\right),
$$

and $\Gamma_{A}$ is

$$
\sum_{n=1,3,5} \Gamma^{i_{1}} \cdots \Gamma^{i_{n}} F_{i_{i} \cdots i_{m}}\left(X^{a^{\prime}}, \theta^{\alpha^{\prime}}, h_{i j k}, a\right) .
$$

Finally the 32-dimensional supersymmetry transformation on the brane is given by the universal eq. (13) with $A\left(X^{a^{\prime}}, \theta^{\alpha^{\prime}}, h_{i j k}, a\right)$ and $C\left(X^{a^{\prime}}, \theta^{\alpha^{\prime}}, h_{i j k}, a\right)$ presented for the M-5-brane above.

The supersymmetry transformations of the bosonic fields, 5 scalars and a tensor, can be obtained using the combination of $\kappa$-symmetry and space-time supersymmetry of these fields and the expression (10). The linearized form of the worldvolume supersymmetry of the $(0,2)$ tensor multiplet was given in [10]. In notation appropriate to a 6-dimensional theory for $\epsilon^{\prime}=0$

$$
\delta_{\epsilon} \theta_{\hat{\beta}}^{s}=\epsilon^{\hat{\alpha} t}\left(\frac{1}{2} \sigma_{\hat{\alpha} \hat{\beta}}^{i}\left(\gamma_{b^{\prime}}\right)_{t}^{s} \partial_{i} X^{b^{\prime}}-\frac{1}{6}\left(\sigma_{\hat{\alpha} \hat{\beta}}^{i j k} \delta_{t}^{s} h_{i j k}\right) .\right.
$$

One can recognize here terms linear and cubic in $\Gamma^{i}$ which form the linear approximation of our matrix $A$.

Note however that the full non-linear action of the self-interacting tensor multiplet has also a symmetry under additional 16-component chiral spinor $\epsilon^{\alpha^{\prime}}=\epsilon_{\hat{\alpha} s}$. The one with the anti-chiral spinor $\epsilon^{\alpha}=\epsilon_{s}^{\hat{\alpha}}$ in the linear approximation relates the spinor of the tensor multiplet to the derivative of scalars and to the tensor field strength. The non-linear action has both chiral as well as anti-chiral supersymmetries.

\section{GENERAL CLASS OF GAUGES}

In general, there is no need to use only the class of gauges defined above which are related to Killing spinors. A much larger class of gauges is available for the quantization of any of the pbranes, D-p-branes and M-branes. This amounts to say that the choice of a projector $\gamma$ can be arbitrary as well as the choice of the gauge which fixes the reparametrization symmetry. In particular, in case of D-p-branes the advantage can be taken from the fact that the quantization consistent with Lorentz symmetry is available [6,2, 2, 3]. In some cases the advantage is to bring the quantized action to a free quadratic action, as in the case of the D-0-brane. This requires a special choice of the gauge for reparametrization symmetry. In generic situation we may formulate here the basic constraint on the choice of the gauge to fix the $\kappa$-symmetry.

Assume that we have chosen the projector $1 \pm \gamma$ and fixed the reparametrization symmetry arbitrarily.

The condition of consistency of this choice can be formulated in a basis where $\gamma$ has 16 of +1 and 16 of -1 on the diagonal.

In this basis the field dependent $\kappa$-symmetry generator $1 \pm \Gamma$ has the following block structure

$1 \pm \Gamma=\left(\begin{array}{cc}1 \pm C & \left(1-C^{2}\right) A^{-1} \\ A & 1 \mp C\end{array}\right)$,

The field dependent matrices $1 \pm \Gamma$ have vanishing determinants and rank 16. For this to happen, the 16-dimensional matrices $1 \pm C$ and $A$ have to 
be invertible. This has to be true in any gauge which is chosen and not only in the Killing gauge, as described above.

For some choice of a projector $\mathcal{P}_{ \pm}$with particular combination of the reparametrization gauge one may encounter a non-invertible $A$ and/or $1 \pm C$. This projector $\mathcal{P}_{ \pm}$together with this choice of the reparametrization gauge can not be used for quantization.

The global supersymmetry on the worldvolume of the 16-component spinor $\theta$ is given by

$\delta \theta=\left(-A\left[(1+C)^{-1}\right]\right)_{g . f} \epsilon+\epsilon^{\prime}$

Here the subscript g.f. means that the field dependent $A, C$ matrices have to be taken at the values of the fields with some particular gaugefixing of reparametrization symmetry.

For example for D-p-branes for $p$ even there is a choice $\gamma=\Gamma_{11}, C=0$ and an invertible $A$ can be find in [6,2,3] together with the total procedure of quantization. This is an example when both $1 \pm C$ and $A$ are invertible for a given choice of $\gamma$. With the same choice of $\gamma$, which gives the only possible Lorentz covariant splitting of the 32 -dimensional spinor in half in $\mathrm{d}=10$, type IIA GS string will have a non-invertible $A=0$ and this gauge is not acceptable as it is known for a very long time. This was always a major obstacle for manifestly supersymmetric Lorentz covariant superstring theory quantization.

\subsection{Covariant Quantization of the D-0- brane}

Consider the $\kappa$-symmetric action of a D-0brane. D-0-brane action does not have BornInfeld field since there is no place for an antisymmetric tensor of rank 2 in one-dimensional theory. The action for $p=0$ case reduces to

$$
S=-T\left(\int d \tau \sqrt{-G_{\tau \tau}}+\int \bar{\theta} \Gamma^{11} \dot{\theta}\right) .
$$

This action can be derived from the action of the massless 11-dimensional superparticle.

$S=\int d \tau \sqrt{g_{\tau \tau}} g^{\tau \tau}\left(\dot{X}^{\hat{m}}-\bar{\theta} \Gamma^{\hat{m}} \dot{\theta}\right)^{2}$,

Here $\hat{m}=0,1, \cdots, 8,9,10$. We may solve equation of motion for $X^{10}$ as $\mathbf{P}_{\hat{10}}=Z$, where $Z$ is a constant, and use $\Gamma^{11}=\Gamma^{\hat{10}}$. From this one can deduce a first order action

$$
\begin{aligned}
S=\int & d \tau\left(\mathbf{P}_{m}\left(\dot{X}^{m}-\bar{\theta} \Gamma^{m} \dot{\theta}\right)\right. \\
& \left.+\frac{1}{2} V\left(\mathbf{P}^{2}+Z^{2}\right)-Z \bar{\theta} \Gamma^{11} \dot{\theta}+\bar{\chi}_{1} d_{2}\right) .
\end{aligned}
$$

The action (36) is invariant under the 16dimensional irreducible $\kappa$-symmetry and under the reparametrization symmetry. The gauge symmetries are (we denote $\Gamma^{m} \mathbf{P}_{m}=\mathbf{P}$ ):

$$
\begin{gathered}
\delta \bar{\theta}=\bar{\kappa}_{2}\left(\Gamma^{11} Z+\mathbf{P}\right), \\
\delta X^{m}=-\eta \mathbf{P}^{m}-\delta \bar{\theta} \Gamma^{m} \theta-\bar{\kappa}_{2} \Gamma^{m} d \\
\delta V=\dot{\eta}+4 \bar{\kappa}_{2} \dot{\theta}+2 \bar{\chi}_{1} \kappa_{2}, \\
\delta \bar{\chi}=\bar{\kappa}_{2} \dot{\mathbf{P}}, \\
\delta d=\left[\mathbf{P}^{2}+Z^{2}\right] \kappa_{2} .
\end{gathered}
$$

Here $\eta(\tau)$ is the time reparametrization gauge parameter and $\kappa_{2}(\tau)=\frac{1}{2}\left(1-\Gamma^{11}\right) \kappa(\tau)$ is the 16 -dimensional parameter of $\kappa$-symmetry. The gauge symmetries form a closed algebra $\left[\delta\left(\kappa_{2}\right), \delta\left(\kappa_{2}^{\prime}\right)\right]=\delta\left(\eta=2 \bar{\kappa}_{2} \kappa_{2}^{\prime}\right)$.

To bring the theory to the canonical form we introduce canonical momenta to $\theta$ and to $V$ and find, excluding auxiliary fields

$L=\mathbf{P}_{m} \dot{X}^{m}+P_{V} \dot{V}+\bar{P}_{\theta} \dot{\theta}$

$+\frac{1}{2} V\left(\mathbf{P}^{2}+Z^{2}\right)+P_{V} \varphi+\left(\bar{P}_{\theta}+\bar{\theta}\left(\mathbb{P}+Z \Gamma^{11}\right)\right) \psi$.

We have primary constraints $\bar{\Phi} \equiv \bar{P}_{\theta}+\bar{\theta} \mathbb{P}+$ $\left.Z \Gamma^{11}\right) \approx 0$ and $P_{V}=0$. The Poisson brackets for 32 fermionic constraints are $\{\Phi, \Phi\}=$ $2 C\left(\mathbb{P}+\Gamma_{11} Z\right)$. We also have to require that the constraints are consistent with the time evolution $\left\{P_{V}, H\right\}=0$. This generates a secondary constraint

$t=\mathbf{P}^{2}+Z^{2}$.

Thus the Hamiltonian is weakly zero and any physical state of the system satisfying the reparamet-

rization constraint is a BPS state $M=|Z|$ since

$\mathbf{P}^{2}+Z^{2}|\Psi\rangle=0 \quad \Longrightarrow$ 


$$
Z^{2}|\Psi\rangle=-\mathbf{P}^{2}|\Psi\rangle=M^{2}|\Psi\rangle .
$$

The $32 \times 32$-dimensional matrix $C\left(\mathbb{P}+\Gamma_{11} Z\right)$ is not invertible since it squares to zero when the reparametrization constraint is imposed. This is a reminder of the fact that D-0-brane is a $\mathrm{d}=11$ massless superparticle. The 32 dimensional fermionic constraint has a 16-dimensional part which forms a first class constraint and another 16-dimensional part which forms a second class constraint. We notice that the Poisson brackets reproduce the $d=10, N=2$ algebra with the central charge which can also be understood as $d=11, N=1$ supersymmetry algebra with the constant value of $\mathbf{P}_{11}=Z$.

We proceed with the quantization and gaugefix $\kappa$-symmetry covariantly by taking $\theta_{2}=0, \theta_{1} \equiv$ $\lambda$ and find

$L_{g . f .}^{\kappa}=\mathbf{P}_{m}\left(\dot{X}^{m}-\bar{\lambda} \Gamma^{m} \dot{\lambda}\right)+\frac{1}{2} V\left(\mathbf{P}^{2}+Z^{2}\right)$.

The 16-dimensional fermionic constraint

$\bar{\Phi}_{\lambda} \equiv\left(\bar{P}_{\lambda}+\bar{\lambda} \mathbf{P}\right) \approx 0$

forms the Poisson bracket

$\left\{\Phi_{\lambda}^{\alpha}, \Phi_{\lambda}^{\beta}\right\}=2(\mathbb{P} C)^{\alpha \beta}$.

The matrix $\mathbf{P C}$ is perfectly invertible as long as the central charge $Z$ is not vanishing. The inverse to $(42)$ is

$\left.\left\{\Phi^{\alpha}, \Phi^{\beta}\right\}^{-1}\right|_{t=0}=\left[2(\mathbf{P} C)^{\alpha \beta}\right]^{-1}=\frac{(\boldsymbol{P})_{\alpha \beta}}{2 \mathbf{P}^{2}}$.

This proves that the fermionic constraints are second class and that the fermionic part of the Lagrangian is not degenerate in a Lorentz covariant gauge. None of this would be true for a vanishing central charge. Note that in the rest frame $\mathbf{P}_{0}=M, \overrightarrow{\mathbf{P}}=0$, hence

$\Phi_{\alpha \beta}=M \delta_{\alpha \beta}$.

For D-0-brane one can covariantly gauge-fix the reparametrization symmetry by choosing the $V=1$ gauge and including the anticommuting reparametrization ghosts $b, c$. This brings us to the following form of the action:

$L_{g . f .}^{\kappa, \eta}=\mathbf{P}_{m} \dot{X}^{m}-\bar{\lambda} \mathbf{P} \dot{\lambda}+\frac{1}{2}\left(\mathbf{P}^{2}+Z^{2}\right)+b \dot{c}$.
Now we can define Dirac brackets

$$
\begin{array}{r}
\{\lambda, \bar{\lambda}\}^{*}=\{\lambda, \bar{\Phi}\}\{\bar{\Phi}, \Phi\}^{-1}\{\Phi, \bar{\lambda}\} \\
=\frac{\mathbf{P}}{2 \mathbf{P}^{2}}=-\frac{\mathbf{P}}{2 Z^{2}} .
\end{array}
$$

The generator of the 32-dimensional supersymmetry is $\bar{\epsilon} Q=\bar{\epsilon}\left(\mathbf{P}+\Gamma^{11} Z\right) \lambda$. It forms the following Dirac bracket

$$
\begin{gathered}
\left.\left[\bar{\epsilon} Q, \bar{Q} \epsilon^{\prime}\right]^{*}=\bar{\epsilon} \mathbb{P}+\Gamma^{11} Z\right) \frac{\mathbf{P}}{2 \mathbf{P}^{2}}\left(\mathbb{P}+\Gamma^{11} Z\right) \epsilon^{\prime}=(47) \\
\left.\bar{\epsilon} \Gamma^{\hat{m}} \mathbf{P}_{\hat{m}} \epsilon^{\prime}=\mathbf{\Phi} \mathbf{P}+\Gamma^{11} Z\right) \epsilon^{\prime} .
\end{gathered}
$$

This Dirac bracket realizes the $\mathrm{d}=11, \mathrm{~N}=1$ supersymmetry algebra or, equivalently, $\mathrm{d}=10, \mathrm{~N}=2$ supersymmetry algebra with the central charge $Z$.

One can also to take into account that the path integral in presence of second class constraints has an additional term with $\sqrt{\operatorname{Ber}\left\{\Phi_{\lambda}, \Phi_{\lambda}\right\}} \sim$ $\sqrt{\operatorname{Ber} \Phi_{\alpha \beta}}$ as it is known from the work of $\mathrm{E}$. Fradkin and collaborators. It can be used to make a change of variables $S_{\alpha}=\Phi_{\alpha \beta}^{1 / 2} \lambda^{\beta}$. The action becomes

$L=\mathbf{P}_{m} \dot{X}^{m}-i S_{\alpha} \dot{S}_{\alpha}+b \dot{c}-H$

$H=-\frac{1}{2}\left(\mathbf{P}^{2}+Z^{2}\right)$.

The generators of global supersymmetry commuting with the Hamiltonian take the form

$\bar{\epsilon} Q=\bar{\epsilon}\left(\mathbb{P}+\Gamma^{11} Z\right) \Phi^{-1 / 2} S$.

Taking into account that $\left\{S_{\alpha}, S_{\beta}\right\}^{*}=-\frac{i}{2} \delta_{\alpha \beta}$ we have again realized $d=10, N=2$ supersymmetry algebra in the form (5.1) or (??).

The terms with anticommuting fields $S_{\alpha}$ can be rewritten in a form where it is clear that they can be interpreted as world-line spinors,

$L=\mathbf{P}_{m} \partial_{0} X^{m}+\bar{S}_{\alpha} \rho^{0} \partial_{0} S_{\alpha}+b \dot{c}-H$.

Here $\bar{S}_{\alpha}=i S_{\alpha} \rho^{0}$ and $\left(\rho^{0}\right)^{2}=-1, \rho^{0}=i$ being a 1-dimensional matrix.

Thus, we have the original 10 coordinates $X^{m}$ and their conjugate momenta $\mathbf{P}_{m}$, and a pair of reparametrization ghosts. There are also 16 anticommuting world-line spinors $S$, describing 8 
fermionic degrees of freedom. The Hamiltonian is quadratic. The ground state with

$$
M^{2}=Z^{2}
$$

is the state with the minimal value of the Hamiltonian. Thus for the D-superparticle one can see that the condition for the covariant quantization is satisfied in the presence of a central charge which makes the mass of a physical state nonvanishing [3]. The global supersymmetry algebra is realized in a covariant way, as different from the light-cone gauge.

Note that the total procedure of quantization has new features due to the fact that we deal with BPS states with the non-vanishing mass. In particular we have divided on $Z^{2}$ at the intermediate level, which would be impossible at $Z=0$. The BPS states with vanishing mass have to be considered separately and with great care, as we know it from the analysis of the massless black holes in string theory [12].

\section{SUMMARY OF NEW RESULTS}

In this talk we have described various new possibilities of quantization of the fermionic worldvolume gauge symmetry, so called $\kappa$-symmetry. Our main focus was on relation between the space-time supersymmetric configurations, or BPS configurations and the new ways of quantization.

It was known for a long time that the superparticle and the superstring with manifest space-time supersymmetry can not be quantized covariantly. Now we have learned that both the D-0-brane and D-1-string as well as all of the D-p-branes do admit covariant gauge-fixing.

We have also used our experience in the properties of the Killing spinors of various BPS solutions in the space-time. We have applied this concept to the quantization of the Green-Schwarz Superstring and Bergshoeff-Sezgin-Townsend Supermembrane. This results in an interesting and yet unexplored non-linear theories of the scalar supermultiplets in $\mathrm{d}=2$ and $\mathrm{d}=3$ dimensions.

One of the most interesting new results is related to the quantization of the M-5brane. The quantization is most fruitful in the fermionic gauge defined by the Killing spinor and reparametrization gauge defined by the Killing vector of the space-time M-5-brane solution. The resulting action defines the non-linear action of the $(2,0) \mathrm{d}=6$ tensor supermultiplet.

\section{REFERENCES}

1. R. Kallosh, Phys. Rev. D52, 6020 (1995).

2. E. Bergshoeff, R. Kallosh, T. Ortín and G. Papadopulos, $\kappa$-symmetry, Supersymmetry and Intersecting Branes, hep-th/9705040.

3. R. Kallosh, Phys. Rev. D56, 3515 (1997), hep-th/9705056.

4. R. Kallosh, Worldvolume Supersymmetry, hep-th/9709069.

5. M. Cederwall, A. von Gussich, B.E.W. Nilsson, and A. Westerberg Nucl. Phys. B490, 163 (1997); M. Cederwall, A. von Gussich, B.E.W. Nilsson, P. Sundell and A. Westerberg, Nucl. Phys. B490, 179 (1997).

6. M. Aganagic, C. Popescu, and J.H. Schwarz, Phys.

Lett. B393, 311 (1997), Nucl. Phys. B495, 99 (1997).

7. E. Bergshoeff and P.K. Townsend, Nucl. Phys. B490, 145 (1997), hep-th/9611173.

8. P. Pasti, D. Sorokin and M. Tonin, Phys. Lett. 398B (1997) 41; I. Bandos, K. Lechner, A. Nurmagambetov, P. Pasti, D. Sorokin and M. Tonin, Phys. Rev. Lett. 78 (1997) 4332.

9. M. Aganagic, J. Park, C. Popescu and J. H. Schwarz, Nucl. Phys. B496 (1997) 191.

10. P.S. Howe and E. Sezgin, Phys. Lett. B394 (1997) 62, hep-th/9611008; P.S. Howe, E. Sezgin and P.C. West, Phys. Lett. B399 (1997) 49, hep-th/9702008; P.S. Howe, N.D. Lambert and P.C. West, The self-dual string soliton, hep-th/9709014.

11. I. Bandos, K. Lechner, A. Nurmagambetov, P. Pasti, D. Sorokin and M. Tonin, On the equivalence of different formulations of the $M$ theory five-brane hep-th/9703127.

12. A. Strominger, Nucl. Phys. B451 (1995) 96, hep-th/9504090. 